\title{
Horizontal axis wind turbine testing at high Reynolds numbers
}

Miller, Mark A.; Kiefer, Janik; Westergaard, Carsten; Hansen, Martin Otto Laver; Hultmark, Marcus

Published in:

Physical Review Fluids

Link to article, DOI:

10.1103/physrevfluids.4.110504

Publication date:

2019

Document Version

Publisher's PDF, also known as Version of record

Link back to DTU Orbit

Citation (APA):

Miller, M. A., Kiefer, J., Westergaard, C., Hansen, M. O. L., \& Hultmark, M. (2019). Horizontal axis wind turbine testing at high Reynolds numbers. Physical Review Fluids, 4(11), [110504].

https://doi.org/10.1103/physrevfluids.4.110504

\section{General rights}

Copyright and moral rights for the publications made accessible in the public portal are retained by the authors and/or other copyright owners and it is a condition of accessing publications that users recognise and abide by the legal requirements associated with these rights.

- Users may download and print one copy of any publication from the public portal for the purpose of private study or research.

- You may not further distribute the material or use it for any profit-making activity or commercial gain

- You may freely distribute the URL identifying the publication in the public portal 


\title{
Horizontal axis wind turbine testing at high Reynolds numbers
}

\author{
Mark A. Miller, ${ }^{1}$ Janik Kiefer, ${ }^{2}$ Carsten Westergaard, ${ }^{3}$ Martin O. L. Hansen, ${ }^{2}$ \\ and Marcus Hultmark ${ }^{1, *}$ \\ ${ }^{1}$ Department of Mechanical and Aerospace Engineering, Princeton University, \\ Princeton, New Jersey 08544, USA \\ ${ }^{2}$ Department of Wind Energy, Technical University of Denmark, \\ DK-2800 Lyngby, Denmark \\ ${ }^{3}$ Westergaard Solutions Inc., Houston, Texas 77006, USA
}

(Received 16 July 2019; published 18 November 2019)

\begin{abstract}
Detailed studies of modern large-scale wind turbines represent a significant challenge. The immense length scales characteristic of these machines, in combination with rotational effects, render numerical simulations and conventional wind tunnel tests unfeasible. Field experiments can give us important insight into the aerodynamics and operation, but they are always accompanied by large amounts of uncertainty, due to the changing nature of the inflow and the lack of accurate control of the test conditions. Here, a series of experiments is presented, using an alternative method that enables us to represent and study much of the physics governing the large-scale wind turbines in small-scale models. A specialized, compressed-air wind tunnel is used to achieve dynamic similarity with the field-scale, but under accurately controlled conditions of the laboratory. Power and thrust coefficients are investigated as a function of the Reynolds number up to $\operatorname{Re}_{D}=14 \times 10^{6}$, at tip speed ratios representative of those typical in the field. A strong Reynolds number dependence is observed in the power coefficient, even at very high Reynolds numbers (well exceeding those occurring in most laboratory studies). We show that for an untripped rotor, the performance reaches a Reynolds number invariant state at $\operatorname{Re}_{c} \geqslant 3.5 \times 10^{6}$, regardless of the tip speed ratio. The same model was also tested with scaled tripping devices, with a height of only $9 \mu \mathrm{m}$, to study the effect of transition on the rotor performance. In the tripped case, the Reynolds number dependence was eliminated for all tested cases, suggesting that the state of the boundary layer is critical for correct predictions of rotor performance.
\end{abstract}

DOI: 10.1103/PhysRevFluids.4.110504

\section{INTRODUCTION}

Modern wind turbine design has been defined by ever-increasing rotor diameters with a tenfold increase over the last four decades. Larger wind turbines mean a reduction in the total number of individual units requiring installation and maintenance, per kilowatt-hour produced, compared to a farm comprised of smaller turbines. The trend toward larger rotors holds whether installation is on- or offshore, with new onshore units exceeding $158 \mathrm{~m}$ and offshore moving past $200 \mathrm{~m}$ [1]. The power that can be extracted from the wind scales linearly with the rotor's swept area, or equivalently with the square of its diameter. Furthermore, a larger wind turbine implies access to higher wind speeds higher up in the atmospheric boundary layer. A wind turbine's ability to convert this available power to useful mechanical power is expressed in the power

\footnotetext{
*hultmark@princeton.edu
} 
coefficient:

$$
C_{p}=\frac{P}{\frac{1}{2} \rho U^{3} \frac{\pi}{4} D^{2}},
$$

where $\rho$ is the air density, $U$ the wind speed, and $D$ the turbine diameter. The measured power output, $P$, of a turbine depends on the fluid density, velocity, viscosity $(\mu)$, speed of sound, as well as specific turbine geometry and operating conditions. The exact nature between these parameters and the power output is not known, which implies that numerical and experimental simulations and modeling are necessary to determine the effect these parameters have on the actual turbine performance. Dimensional analysis can inform us that the aerodynamics of a given turbine design is a function of three other nondimensional parameters. These three parameters are the Reynolds number, the tip speed ratio, and the Mach number, which describe the relative effect of viscosity to the inertia of the fluid, the effects of rotation on the flow field, as well as compressibility effects, respectively.

While these nondimensional parameters govern the overall rotor response, added complexities in the atmosphere, such as turbulence, wind shear, and buoyancy, as well as structural properties further complicate the problem. For example, Herges et al. [2] show a remarkable difference between unstable and stable atmospheric conditions. One of the most common methods for quantifying turbine performance is through field measurements of the power coefficient. This method is complicated by the constantly changing, and challenging to monitor, inflow conditions present at any field site. Factors such as temperature, static pressure, wind shear, turbulence, and wind direction must all be constantly monitored and quantified, as no steady-state conditions exist in the field [3].

Power curves must then be collected over long periods of time, typically requiring months before acceptable convergence is observed in the statistics [4]. Therefore, although important as a validation tool, field experiments are both difficult to perform and expensive to implement while having a large amount of uncertainty in the results. This has driven a decades-long effort by wind turbine manufacturers and researchers to develop engineering design tools and modeling techniques for predicting turbine performance. The reliance on these modeling methods has a long history, with the basic design tool for aerodynamic input loads [known as blade element momentum (BEM)] remaining essentially the same since being developed in 1935 by Glauert $[5,6]$. It is widely used in industry due to its low computational cost and the extensive legacy of modeling, validation, and certification efforts upon which most of the current iterations of the method are based. However, BEM has a number of inherent deficiencies that must be accounted for through various engineering-type models. Some of the more common corrections attempt to capture three-dimensional and rotational effects, high thrust loading, yawed and turbulent inflow, and many other conditions commonly encountered in the field. These corrections are essential to model the loads accurately, and as such remain an active area of research. One downside of such methods is that limited additional insight can be gained into off-design conditions commonly encountered in the field, such as unsteady or time-varying loads, forcing designs to remain relatively conservative to meet the certification and lifetime requirements of the modern turbine.

The dilemma of BEM and field testing has long been known to the research community, and the lack of experimental validation cases was cited as one of the biggest research challenges in a recent review article by van Kuik et al. [7]. Attempts at reducing the uncertainty in the power coefficient measurements have been made in two parallel tracks, via numerical simulations using the actuator-line method and laboratory experiments. The challenge for both approaches lies in the large physical scale of the turbine units themselves. This implies that the Reynolds number is very high, which in turn implies that the flows are turbulent:

$$
\operatorname{Re}_{D}=\frac{\rho U D}{\mu}
$$


Values of $\operatorname{Re}_{D}$ range from $O\left(10^{5}\right)$ for small-scale turbines with $D \approx 1-10 \mathrm{~m}$ up to $O\left(10^{8}\right)$ for the largest offshore units with $D \geqslant 200 \mathrm{~m}$. Unfortunately, the high Reynolds numbers make direct numerical simulations (DNSs), where the governing equations are numerically solved at all length and time scales, unfeasible. Instead, large eddy simulation (LES), Reynolds-averaged Navier-Stokes simulations (RANs), or inviscid/vortex-method solvers are used for studying wind turbine flows, where some or all of the turbulence is modeled within the solver. Additionally, except for all but the most advanced LES methods, the flow over the blades and rotors is not resolved, but instead modeled with a distributed forcing on the local numerical grid, in a BEM-like fashion, which requires two-dimensional (2D) airfoil data as input, meaning that 3D and unsteady effects are not directly captured with these methods either.

From an experimental standpoint, few studies have been performed at what can reasonably be considered full-scale Reynolds number values. The underlying issue is the presence of the two nondimensional parameters in addition to the Reynolds number. The tip speed ratio and Mach number are defined as

$$
\lambda=\frac{\omega R}{U}, \quad \mathrm{Ma}=\frac{\omega R}{a}
$$

with $\omega$ the angular velocity and $a$ the sound-speed. Fundamentally, an experimental study seeks to operate with a much smaller diameter than the full scale, typically two orders of magnitude or more. This means that if the tests are conducted in conventional wind tunnels, the free-stream velocity must be increased by approximately 100 times or more compared to the field value, to match $\mathrm{Re}_{D}$. Altering velocity has a dominolike effect on the other nondimensional parameters, causing $\lambda$ to decrease unless $\omega$ is increased to the rotor scale-ratio squared, or more than four orders of magnitude. Mechanically, this is not feasible due to the centrifugal forces created, and it also creates significant compressibility effects, as can be seen in Eqs. (3). For these reasons, many laboratory experiments are performed at reduced Reynolds numbers [8], with only two notable exceptions where the models were physically large (4-10 $\mathrm{m}$ in diameter) and very large wind tunnels were utilized [3,9]. These studies have provided a number of insights into turbine operation at the full scale, among them disparities between the BEM and measured loads [3], also a clear indication of Reynolds number effects despite the large $\operatorname{Re}_{D}$ tested [10], and even some disparities between the rotationally driven performance augmentation of both studies [11]. While these works provided some of the clearest insight yet into full-scale operation, there is still a definitive need for additional work concerning Reynolds number effects on turbine performance.

An additional, complicating issue is that experiments (even those performed at large scale) are typically operated with fixed $\omega$, while the tunnel velocity is altered to affect the tip speed. This means that rotational effects and Reynolds number effects are intermixed, as is also the case in field operation where $\omega$ can only vary by a small amount (due to restrictions on the generator, while blade pitch is used to control shaft power). A truly variable speed wind turbine model is required to extensively characterize both Reynolds number and tip speed ratio effects in detail. The lack of prior experimental work in this area again points to a need for additional quantification of these effects in a manner that independently lends additional physical insight to the problem of scaling behavior for wind turbines.

In this study, we aim to address the question of performance scaling with Re and $\lambda$ for horizontal axis wind turbines (HAWTs). The challenges associated with wind tunnel tests, as discussed above, have been bypassed by implementing a specialized wind tunnel that uses highly compressed dry air as the working fluid. Increasing the pressure up to 238 bar allows for working fluid densities of over 200 times that of air at atmospheric pressure. Operating at variable densities effectively decouples the Reynolds number from the tip speed ratio, as the density only enters via the Reynolds number. This allows high Reynolds numbers to be achieved without the need for high tunnel velocities; instead they are on the same order as in the field, which avoids compressibility effects and keeps the timescales relatively large. In this context, Reynolds number and tip-speed-ratio effects can be 


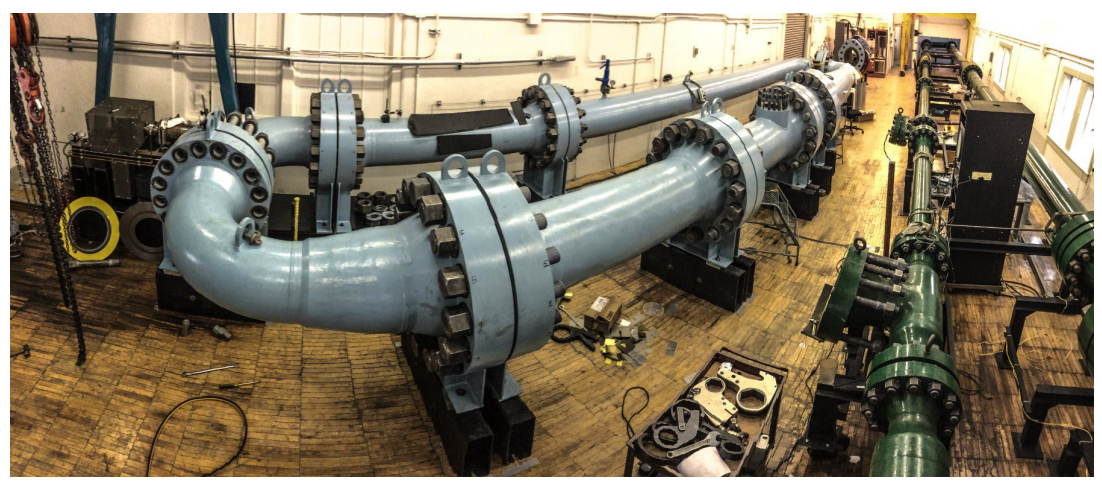

FIG. 1. The High Reynolds number Test Facility (HRTF, in light blue) located at the Princeton Gas Dynamics Laboratory.

characterized independently of each other, and without Mach number effects or any assumptions or modeling.

\section{EXPERIMENT DESCRIPTION}

The experimental facility used in this work is a specialized, compressed air wind tunnel known as the High Reynolds number Test Facility (HRTF). It is a recirculating-type, low-velocity, high static pressure wind tunnel that uses air as the working fluid. The facility can support up to $24 \mathrm{MPa}$, and free-stream velocities in the test section of up to $10 \mathrm{~m} / \mathrm{s}$. The test section itself is $4.88 \mathrm{~m}$ long with an inside diameter of $48.9 \mathrm{~cm}$. This gives a blockage ratio (using the turbine swept area) of $A_{\text {model }} / A_{\text {tunnel }}=16.7 \%$ for the $20 \mathrm{~cm}$ rotor. Upstream of the test section is a contraction with an area ratio of 2.43:1 in which a series of flow conditioning screens and honeycomb straighteners are located. Free-stream velocity is measured via a pitot-static tube located upstream of the turbine model using a differential pressure transducer with a range of $3447 \mathrm{~Pa}$ (Validyne DP-15). This facility has been used previously to study wakes of axisymmetric bodies [12-14], zero-pressure gradient turbulent boundary layers [15], and more recently various wind turbine models of both vertical and horizontal axis varieties [16-18]. The HRTF is shown in Fig. 1.

In the pressurized test environment, real-gas effects are accounted for via

$$
\rho=p_{\mathrm{s}} /\left(Z R_{a} T\right)
$$

where $R_{a}$ is the specific gas constant for air, $T$ is the tunnel temperature, and $Z$ is the compressibility factor. For dry air, $Z$ changes by only $10 \%$ for static pressures in the range 1-233 bar, meaning that density changes nearly linearly with static pressure in the test section. For all experimental data sets, the exact density and viscosity of the compressed air are calculated using the real-gas relationship of Eq. (4) with measurements of $p_{\mathrm{s}}$ and $T$ from the test section (this method is outlined in Ref. [19]). The key to high Reynolds number testing using compressed air is that both dynamic viscosity and sound speed are only very weak functions of pressure, meaning that free-stream velocity can remain relatively low and the $\omega$ required for $\lambda$ matching is mechanically feasible.

\section{A. Wind turbine model}

Challenges of operating in a pressurized environment include large forces and torques acting on the model, which scale linearly with fluid density. For instance, a model tested at the maximum HRTF pressure will see loads that are in excess of 200 times compared to those experienced in an atmospheric wind tunnel at the same velocity. Careful consideration was given to model design so as to minimize rotor deflections during even the most extreme operating conditions. 
TABLE I. Wind Turbine Model Geometry. Between sections 1 and 2, the geometric shape transitions from a circular hub to an airfoil shape. The relative twist of these intermediate sections is set to $13^{\circ}$.

\begin{tabular}{lcccc}
\hline \hline Section & Radius $(\mathrm{mm})$ & Chord $(\mathrm{mm})$ & Twist & Airfoil \\
\hline 1 & 3.704 & 11.11 & $13^{\circ}$ & Circular \\
2 & 14.815 & 28.89 & $13^{\circ}$ & NACA 63-235 \\
3 & 100.0 & 11.11 & $0^{\circ}$ & NACA 63-214 \\
\hline \hline
\end{tabular}

The rotor model used in these experiments is $20 \mathrm{~cm}$ in diameter and attaches to the tower gearbox via a threaded M7 hub. In this series of experiments, the rotor solidity is increased in order to delay the stall tip speed ratio as well as increase the blade Reynolds number and torque. However, the current model operates in the same fashion as a full-scale, slender unit, such as the observed tip speed ratios, and power/thrust coefficients are reasonable (i.e., in the lift-producing regime). Lower solidity would likely push the operational tip speed ratio range to larger values and the resulting lower Reynolds numbers (based on chord length) would probably increase the transition effects observed in this study. Future investigations will include studies of the effect of solidity, and allow for investigations of rotors more similar to modern field turbines.

Geometrical details of the three driving sections at hub, root, and tip locations are given in Table I. The airfoil geometries, chord, thickness, and twist distribution between the sections were input to a computer-aided design (CAD) program. For sections $2-3$, the extrapolation is straightforward since the airfoils are of the same family, and only the twist, chord, and thickness should be altered. Near the hub, the extrapolation between sections 1 and 2 is more complicated due to the circular hub geometry. It is expected that this has little effect on the performance, however, since it only comprises the inner $15 \%$ of the rotor. Three-dimensional CAD files are available upon request if additional information is needed regarding geometrical features of the rotor.

In general, the twist axis of the rotor was located at the quarter-chord of the airfoils, with the exception of the circular hub at section 1 where the twist axis was at the center of the circle. The twist schedule between sections 2 and 3 is nonlinear and was determined with the following equation:

$$
\text { Twist }\left(^{\circ}\right)= \begin{cases}13 & \text { if } r / R \leqslant 4 / 27, \\ 18.148\left(\frac{R-r}{R}\right)^{3}-7.698\left(\frac{R-r}{R}\right)^{2}+8.504\left(\frac{R-r}{R}\right) & \text { otherwise. }\end{cases}
$$

Similarly, the thickness has a linear distribution between sections 1 and 2 , but is nonlinear between 2 and 3, as given by Eq. (6). The distribution of both the twist and thickness schedules are shown graphically in Fig. 2(a),

$$
\text { Thickness }(\%)= \begin{cases}100 & \text { if } r=0, \\ 585.01 \frac{R-r}{R}-463.34 & \text { if } 0<r / R \leqslant 4 / 27 \\ 27.42\left(\frac{R-r}{R}\right)^{3}-61.41\left(\frac{R-r}{R}\right)^{2}+57.07\left(\frac{R-r}{R}\right)+14 & \text { otherwise. }\end{cases}
$$

Meanwhile, the chord schedule between locations 2 and 3 is purely linear for any section falling between that given in Table I. Rotor pitch is fixed at $5^{\circ}$ defined as positive into the oncoming flow from the rotor plane. Yaw, cone, and teeter angle have all been set to $0^{\circ}$. The spinner hub geometry was taken directly from Reynolds [20], and it consists of a NACA 1 series spinner scaled to the appropriate size for the model. The final rotor is CNC milled from a solid block of aluminum alloy, which ensures a high tolerance on the surface finish and dimensional accuracy. The final model is shown in Fig. 2(b). Particular care was given to the surface finish of the model as matching all length scales is required for full-dynamic similarity. The surface roughness was carefully measured using an Olympus LEXT OLS4000 confocal microscope, and the area-averaged, root-mean-square roughness height was found to be $S_{q} \leqslant 800 \mathrm{~nm}$ consistently among all three blades. The effect of surface condition on observed rotor performance is discussed in Sec. VI. Section V B details the 


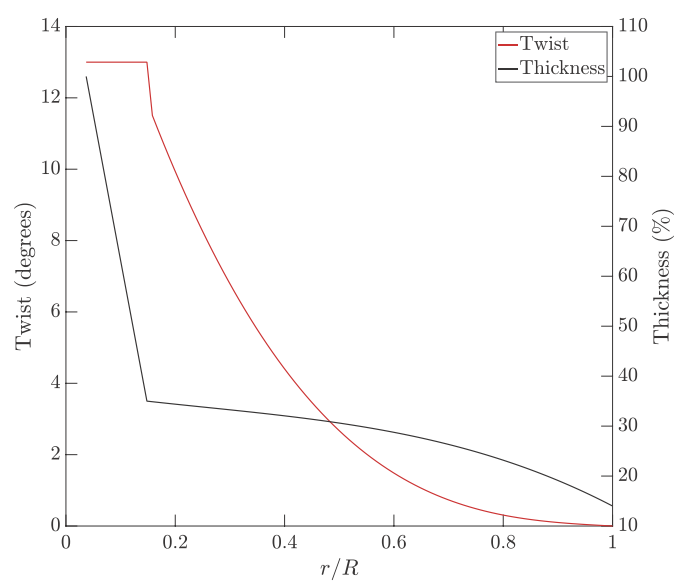

(a)

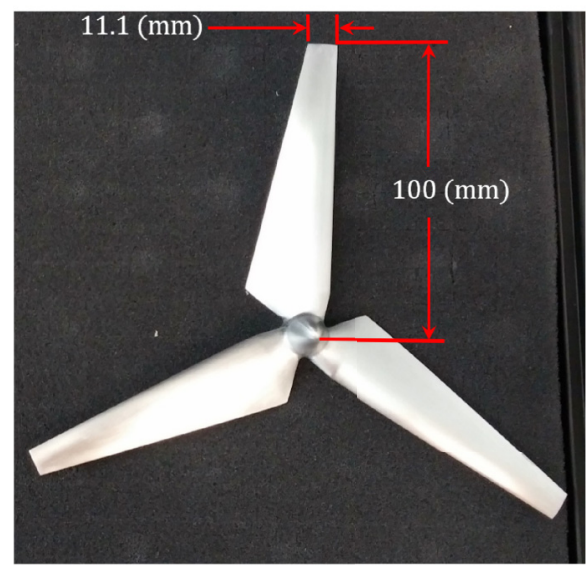

(b)

FIG. 2. The twist and thickness schedule for the rotor are shown in plot (a). The finished model rotor is given by the image in (b).

addition of prescribed tripping devices designed with this same methodology to force transition on the rotor blade surface.

The model tower and nacelle were designed to handle the high loading cases present at maximum tunnel density inside the HRTF. Their design and construction is detailed in Ref. [16]. The tower cross section resembles a diamond-shape with rounded edges, so as to support the large aerodynamic thrust and torque imposed by a spinning rotor with minimal deflection and additional drag. Details concerning blockage effects due to the tower and rotor are discussed in Appendix.

\section{B. Instrumentation and turbine operation}

A measurement and control stack was designed to accurately resolve the mechanical loads produced by the model turbine. Shaft torque, $\tau$, angular velocity, $\omega$, force components at the tower base $\left(F_{x}, F_{y}\right.$, and $\left.F_{z}\right)$, as well as moments at the tower base $\left(M_{x}, M_{y}\right.$, and $\left.M_{z}\right)$ are measured simultaneously during an experimental campaign. The physical layout of the model and measurement stack starts with the solid rotor model of fixed pitch as described in Sec. II A. The model is mounted on the input shaft of the tower. Inside the tower itself is a small right-angle bevel gearbox which redirects the mechanical power $90^{\circ}$ and outside of the tunnel test section for measurement. Correction methodologies that account for gearbox inefficiencies are discussed in Appendix 1. A six-axis force and moment transducer (JR3 Inc. model 75E20A4) is mounted at the base of the tower for measurement of the aforementioned forces and moments on the tower. The force sensor body is bolted to a rotary table, allowing for adjustment of the turbine yaw. The output shaft for the tower gearbox is located in the center of the measurement stack and passes through the central axis of the force sensor and rotary table. The output shaft is then coupled to a torque transducer (Magtrol model TM-305 with a range of $\pm 2 \mathrm{Nm}$ ). This unit allows for time-resolved measurements (temporal resolution of $5 \mathrm{kHz}$ ) of the torque signal and determination of the rotational speed via an internal optical encoder. Power is dissipated from the system using a magnetic hysteresis brake (Magtrol model AHB-3), which applies precise shaft torque loads; giving full control over $\omega$ independently of wind speed and density. The brake serves the same function as the generator of a full-scale turbine, with greatly increased accuracy and flexibility in its operating window. All of these components together make up the measurement stack, shown in Fig. 3. The uncertainties associated with each measurement device are summarized in Table II. 


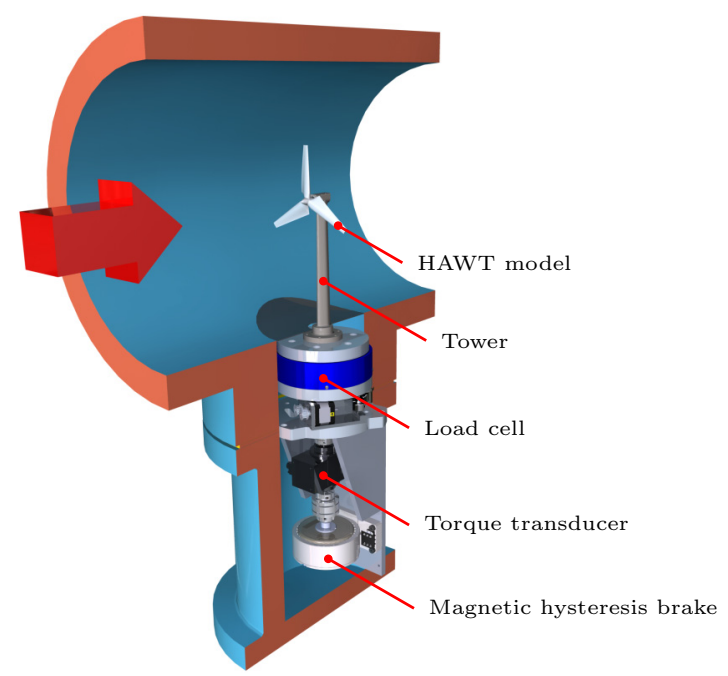

FIG. 3. Sectional view of the HRTF test section with the model wind turbine and measurement stack in place. The red arrow shows the direction of flow.

\section{EXPERIMENTAL PROCEDURE}

The HAWT model used in these experiments is operated in a manner similar to that of full-scale modern wind turbines with full variable speed drives [21]. The experiments presented here can easily be operated at many different rotation rates as all the mechanical energy is dissipated as heat by the magnetic hysteresis brake, which is passively cooled by the high density air. This method is in principle a full variable-speed wind turbine, where any $\omega$ may be specified during operation. Field data are often viewed in terms of the inflow velocity $U$, since $\omega$ is fixed. This creates uncertainty when viewing data plotted as a function of $\lambda$, as wind and rotor speed correlation is only obtained through the 10 min binning discussed earlier. Another issue with this method of viewing data is that not only is $\lambda$ changing, but $\operatorname{Re}_{D}$ is additionally being altered along the abscissa, making it inherently difficult to separate these two effects in the field when plotting data as a function of tip speed. For the experiments presented here, we have elected to keep $U$ and $\rho$ approximately constant during a run, and we vary $\omega$ over a predetermined range. This means that for a given measured $C_{p}$ or $C_{t}$ curve, the inflow conditions, and hence $\operatorname{Re}_{D}$, remain constant while $\lambda$ is altered. In this way, Reynolds number effects and tip-speed-ratio effects can directly and independently be observed and studied. Operating the model turbine with fixed inflow additionally allows for convenient data collection. If the model were to be operated as in the field, an additional controller would be needed to maintain a fixed rotation rate as the tunnel velocity or density is altered. If field-style data are required, they may

TABLE II. Experimental uncertainty sources listed include linearity, hysteresis, and temperature influences combined in a root-mean-square sense for each sensor.

\begin{tabular}{lcc}
\hline \hline Measurement unit & Symbol & Total uncertainty \\
\hline Torque signal & $\tau$ & $u_{\tau}= \pm 0.003(\mathrm{Nm})$ \\
Angular velocity & $\omega$ & $u_{\omega}= \pm 0.105(\mathrm{rad} / \mathrm{s})$ \\
Axial thrust force & $F_{t}$ & $u_{F_{t}}= \pm 2.5(\mathrm{~N})$ \\
Pitot-static pressure transducer & $\bar{p}$ & $u_{\bar{p}}= \pm 34(\mathrm{~Pa})$ \\
Density & $\rho$ & $u_{\rho}= \pm 0.36 \%$ \\
Viscosity & $\mu$ & $u_{\mu}= \pm 0.8 \%$ \\
\hline \hline
\end{tabular}


be interpolated from the finely resolved measurements at the desired inflow conditions. Dynamic similarity implies that for a geometrically similar rotor, when $\operatorname{Re}_{D}$ and $\lambda$ are matched, all other Reynolds numbers such as $\operatorname{Re}_{c}$, or however Re is defined, are matched as well.

The following sections explore the power-output and resulting thrust forces on the HAWT model. In the experiments presented herein, a small-scale model is tested with Reynolds number, tip speed ratio, and Mach numbers that simultaneously match those experienced by full-scale wind turbines. The primary goal of this work is to gain quantitative insight into the effects of Reynolds number on the performance of modern-day wind turbines. Furthermore, it sets the stage for a long list of questions that can be answered using the same methodology. The set of experiments presented here have been restricted to what can be considered the most canonical case for a wind turbine, where the inflow is steady, uniform, and laminar, the rotor plane is aligned perpendicular to the inflow velocity vector, and the free-stream conditions are fixed for each power curve (i.e., $\operatorname{Re}_{D}$ constant during a run). A significant amount of consideration has also been given to account for experimental uncertainties in the drive-train and for blockage effects, as detailed in Appendix A. All of these methods have enabled an unprecedented level of precision in the data acquisition, and allowed for a detailed investigation of $\operatorname{Re}_{D}$ and $\lambda$ effects.

\section{UNTRIPPED HAWT ROTOR PERFORMANCE WITH REYNOLDS NUMBER}

Performance of the wind turbine model is characterized using the nondimensional power coefficient as given by Eq. (1) and the thrust coefficient:

$$
C_{t}=\frac{F_{t}}{\frac{1}{2} \rho U^{2} A},
$$

where $F_{t}$ is the streamwise force exerted on the hub by the rotor (commonly referred to as the thrust force). Together, $C_{p}$ and $C_{t}$ indicate the forcing placed onto the flow and therefore drive wake structure and dynamics. Furthermore, they are typically used as the defining performance metrics during the design phase, with $C_{p}$ in particular being directly tied to the projected revenue of a turbine. In the context of these experiments, careful measurements have been made of both parameters so that conclusions may be drawn as to their dependence on both tip speed ratio and Reynolds number. The following section describes the method of data validation used for these experiments. Then Reynolds number trends are discussed for the untripped rotor. The same rotor is then studied with the transition location fixed near the leading edge along the span via the use of an array of miniature tripping devices.

\section{A. Experimental data validation}

The unique nature of the HRTF allows for adjustment of the tunnel density independently of the free-stream velocity to achieve a given value of the Reynolds number. Utilizing this capability, a specific Reynolds number case can be achieved using various combinations of operating conditions. As such, different combinations of velocity, density, and rotational speed can result in exactly the same Reynolds number and tip speed ratio and thus yield identical values of the power and thrust coefficient. For all possible operating conditions, the free-stream Mach number is always low enough to neglect compressibility effects. This is not only an excellent method to validate the experimental results, it also enables significant reduction of measurement uncertainty since the forces and torques can be tailored to the instrumentation. Figure 4 highlights this unique feature where different operating conditions together yield a mean Reynolds number of $\operatorname{Re}_{D}=$ $5.099 \times 10^{6} \pm 34000$. The bounds on $\mathrm{Re}_{D}$ reflect the maximum deviation of any given data set from the mean Reynolds number value due to slight variations in tunnel conditions from run-to-run. Tunnel density is shown in the legend of Fig. 4(a) [with the legend also applying to Fig. 4(b)]. Note that despite very different densities between the data sets, the power curves collapse well within the experimental uncertainty when nondimensionalized in Fig. 4(b) with the shaded bars 


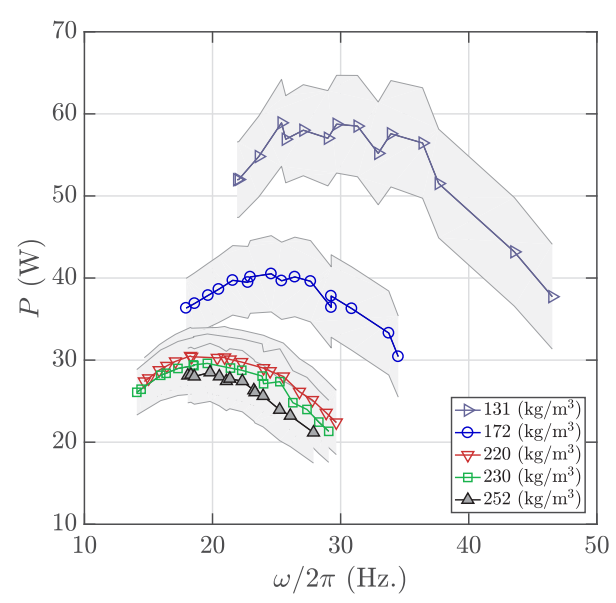

(a)

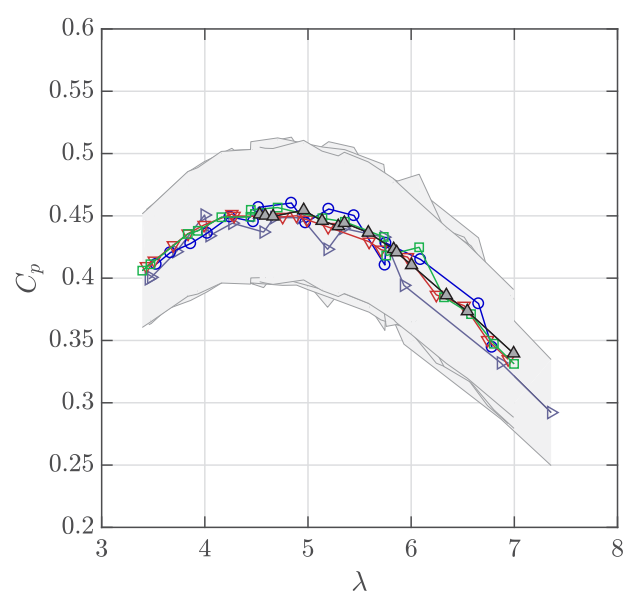

(b)

FIG. 4. Dimensional plots in (a) show measured power vs the rotational frequency at a matched Reynolds number of $\operatorname{Re}_{D}=5.099 \times 10^{6} \pm 34000$. Note that the data set at $\rho=252 \mathrm{~kg} / \mathrm{m}^{3}$ (with shaded symbols) were taken with a different gearbox. The plot to the right in (b) shows the same data nondimensionalized by the free-stream conditions. The shaded area represents experimental uncertainty.

giving the uncertainty calculated at each operating point (details of the uncertainty analysis are given in Table II). Collapse is seen across all cases in $C_{p}$, with slightly more scatter in the $\rho=131 \mathrm{~kg} / \mathrm{m}^{3}$ case, which may be due to the increased rotational rate of the lower-density, higher free-stream velocity of this run. A single, high-density case using a different gearbox at $\rho=252 \mathrm{~kg} / \mathrm{m}^{3}$ is also given and agrees well with the other three data sets, providing validation for the correction methodology used for the gearbox (as discussed in Appendix). The experimental data were validated using the above methodology at several Reynolds numbers $\left(\operatorname{Re}_{D}=7 \times 10^{6}\right.$, $10 \times 10^{6}$, and $12 \times 10^{6}$ ). The resulting nondimensional power and thrust are shown in Fig. 5. The rightmost column of plots of Fig. 5 shows the thrust coefficient, which displays more scatter than the power coefficient, especially at higher Reynolds numbers of $10 \times 10^{6}$ and $12 \times 10^{6}$. This is primarily due to the larger uncertainty associated with the load cell used for axial force measurement (details in Sec. II B). The power coefficient shows excellent collapse across all tested Reynolds numbers and gearboxes used for these measurements. As the Reynolds number is increased, the signal-to-noise ratio also increases due to the larger loads measured. This has the direct effect of reducing the uncertainty as $\mathrm{Re}_{D}$ is increased, which is reflected by the shrinking error-bars shown in gray. As mentioned earlier, each case can be tailored to maximize the measurement range of the sensors, thus reducing the uncertainty. For example, at low Reynolds number, combining lower density with higher velocity yields larger forces and torques. Such optimization of the operating conditions is not possible in an atmospheric-pressure facility, where instead larger uncertainty often must be accepted, or different instrumentation must be used for each test case.

\section{B. Untripped rotor performance with Reynolds number}

The power and thrust coefficients are shown in Fig. 6 for a range of $\operatorname{Re}_{D}$ values from 4 to 14 million. The power coefficient shows a clear dependence on Reynolds number, with the lowest $\operatorname{Re}_{D}$ case tested giving the highest power coefficient and a clear trend where the power coefficient is reduced as Reynolds number is increased. This observation is quite unexpected, as prior literature near these $\operatorname{Re}_{D}$ values has reported the opposite trend [10], although the specific airfoils used for these two rotors are different. It is further observed that the power coefficient levels off and is starting 


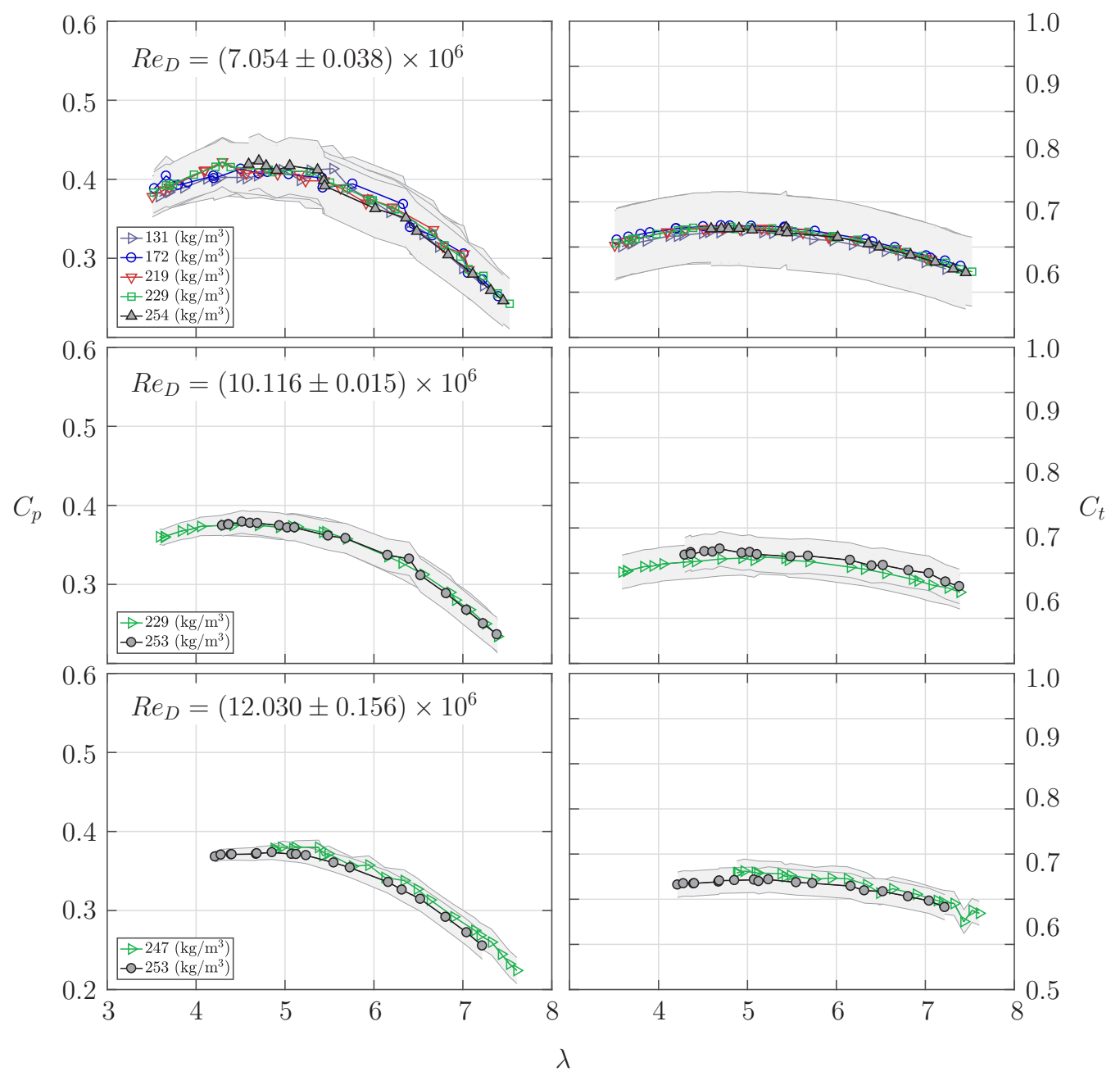

FIG. 5. Power and thrust coefficient data validation for three different Reynolds numbers. Labels for the Reynolds number and the legends apply to each pair of horizontal plots. Data sets with shaded symbols used a different gearbox.

to exhibit collapse at higher Reynolds numbers. This implies that for the specific rotor geometry and inflow conditions used here, above a critical $\mathrm{Re}_{D}$ value the power coefficient is independent of Reynolds number. The results on the thrust coefficient are less pronounced with no clear trend evident with Reynolds number, although strong conclusions cannot be made at this point, as the data fall within the experimental uncertainty. However, it is clear that if a trend exists with the Reynolds number it is much less pronounced compared to that in the power coefficient.

Results from the power coefficient indicated a relatively large $\operatorname{Re}_{D}$ value was necessary for invariance to the Reynolds number. However, higher values of $\lambda$ appear to show invariance at lower $\operatorname{Re}_{D}$. For instance, at $\lambda=7$, invariance occurs around $\operatorname{Re}_{D} \geqslant 8 \times 10^{6}$, whereas at $\lambda=5$ a $\operatorname{Re}_{D} \geqslant$ $10 \times 10^{6}$ is required. Such a two-parameter dependence indicates that neither parameter independently captures the physical mechanisms at play. Traditionally, aerodynamics is parametrized with the Reynolds number based on the chord length and the relative velocity [8]. Such parametrization is quite inconvenient in this case as the chord Reynolds number varies throughout the rotor. However, 


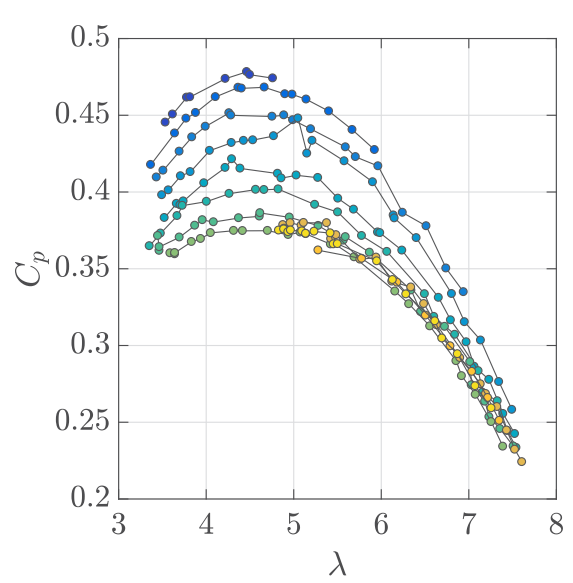

(a)

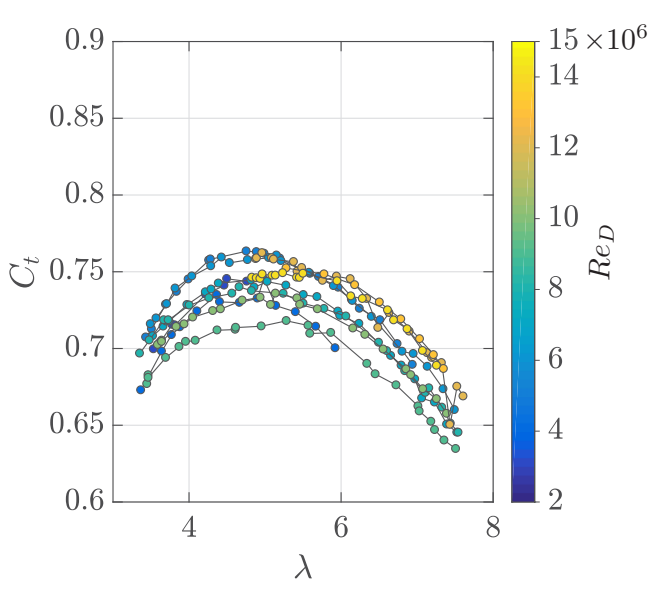

(b)

FIG. 6. Reynolds number trends of the untripped rotor for power (a) and thrust (b) coefficients. The colorbar on the right represents the Reynolds number based on the rotor diameter for both plots, and it is held constant for each measured curve.

the physical effects described by the chord Reynolds number can be captured by a combination of $\operatorname{Re}_{D}$ and $\lambda$ and, for example, the chord length along the blade. This results in a nondimensional group from easily measured and reproducible quantities. Here, a Reynolds number based on the tip geometry has been utilized that may be determined if $\mathrm{Re}_{D}, \lambda$, and the ratio of tip chord to diameter are known as

$$
\operatorname{Re}_{c}=\frac{\rho c \sqrt{U^{2}+(\omega R)^{2}}}{\mu}=\operatorname{Re}_{D} \frac{c}{D} \sqrt{1+\lambda^{2}} .
$$

The parameter $\operatorname{Re}_{c}$ represents the maximum possible Reynolds number that could be encountered at the rotor tip, and as such the true chord Reynolds number is slightly lower. Furthermore, experimental data from many sources can be couched in terms of this definition of the Reynolds number if only a few measured quantities are known and no modeling is necessary to determine $\operatorname{Re}_{c}$. The current data were not acquired at predetermined values of the tip speed ratio, and so the data presented in Fig. 6, along with data from additional cases, have been interpolated to a fixed $\lambda$ grid. This makes calculating $\operatorname{Re}_{c}$ straightforward for all data points. The power coefficient as a function of $\operatorname{Re}_{c}$ is shown in Fig. 7(a) for various values of the tip speed ratio.

Individual curves in this figure represent the fixed $\lambda$ grid values to which the measured power coefficient was interpolated. Moving up in $\operatorname{Re}_{c}$ [i.e., across the abscissa of Fig. 7(b)] is equivalent to fixing $\lambda$ and traveling vertically downward in the power curves of Fig. 6(a). Whenever a power curve is crossed, the local value of $C_{p}$ is interpolated and the resulting value of $\operatorname{Re}_{c}$ is calculated. Since the power curves are relatively dense with data points (typically consisting of more than 15 individual tests), errors due to this interpolation are expected to have a minimal effect on the results. In this way, the rate at which the power curves approach the high Reynolds number limit can be directly observed. The resulting plot gives the power coefficient as a function of the blade Reynolds number and is shown in Fig. 7(a).

Neglecting the $\lambda=3.5,4.0$, and 4.5 cases due to the low maximum values of $\operatorname{Re}_{c}$, the remaining curves show a plateaulike behavior above $\operatorname{Re}_{c}=3.5 \times 10^{6}$ whereby any additional increases in $\operatorname{Re}_{c}$ do not appear to affect the power coefficient of the turbine. This suggests that $C_{p}$ has become invariant to the Reynolds number above this limit. Using the data of Fig. 7(a), the invariant power coefficient can then be directly calculated by averaging power coefficient values that fall above the threshold of $\operatorname{Re}_{c} \geqslant 3.5 \times 10^{6}$. The resulting parameter is denoted as $C_{p, \infty}$ and is shown in 


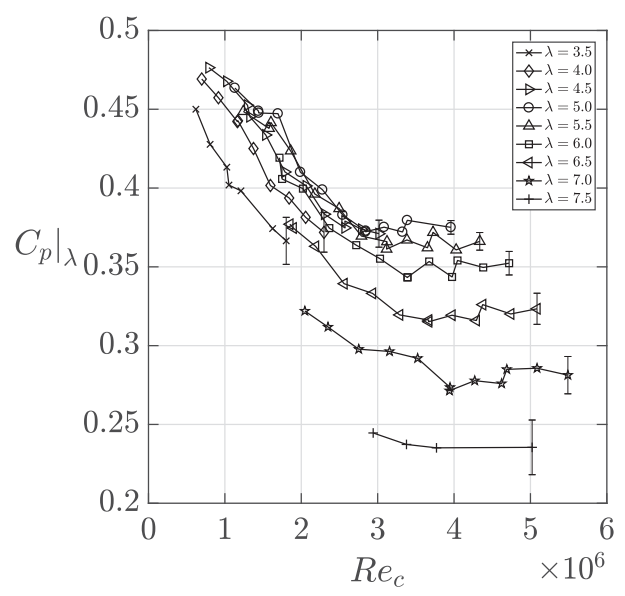

(a)

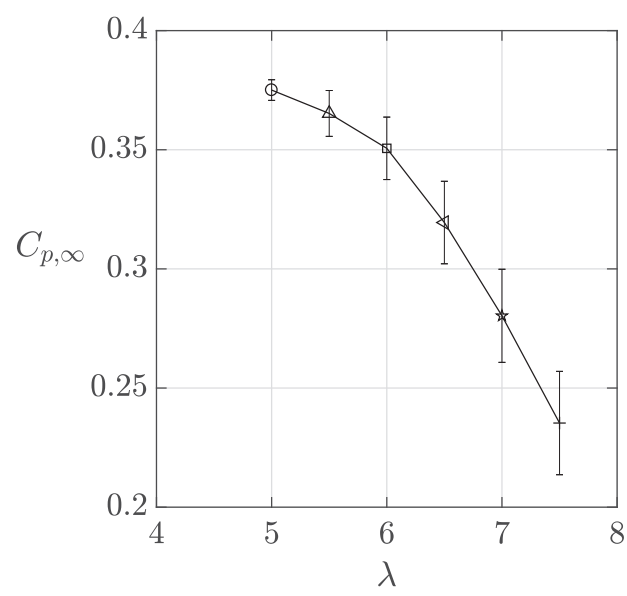

(b)

FIG. 7. Power coefficient as a function of the local blade Reynolds number for a variety of fixed tip speed ratios in (a). Part (b) shows the Reynolds number invariant power curve for the model turbine geometry used under laminar inflow conditions. This plot was created by finding the mean value of $C_{p}$ at fixed $\lambda$ when $\operatorname{Re}_{c} \geqslant$ $3.5 \times 10^{6}$.

Fig. 7(b). This is the power curve that would be returned by a model or field-scale turbine of the same geometry when $\operatorname{Re}_{c} \geqslant 3.5 \times 10^{6}$. Such a Reynolds number invariant power curve can be used as a reference point for numerical simulations or model building studies, and to evaluate Re behavior of other experiments using the same geometry.

Further insight can be gained into the behavior of the rotor aerodynamics at lower Reynolds number by combining the results of Figs. 7(a) and 7(b). For each tip speed ratio, the entire curve of Fig. 7(a) is normalized by the relevant $C_{p, \infty}$ value of Fig. 7(b). This is shown in Fig. 8. If the cutoff Reynolds number has been chosen correctly, all curves will trend to unity, with some scatter due to experimental uncertainty as can be seen in the figure. An expected collapse is seen across all tip speeds for lower $\operatorname{Re}_{c}$ values as well. This further indicates that the parameter $\operatorname{Re}_{c}$ accurately characterizes the physics responsible for the lower Reynolds number augmentation. This implies that the performance of the turbine at reduced Reynolds numbers can be found with a limited number of experiments. For example, if the invariant power curve is known, then experiments at a single $\lambda$

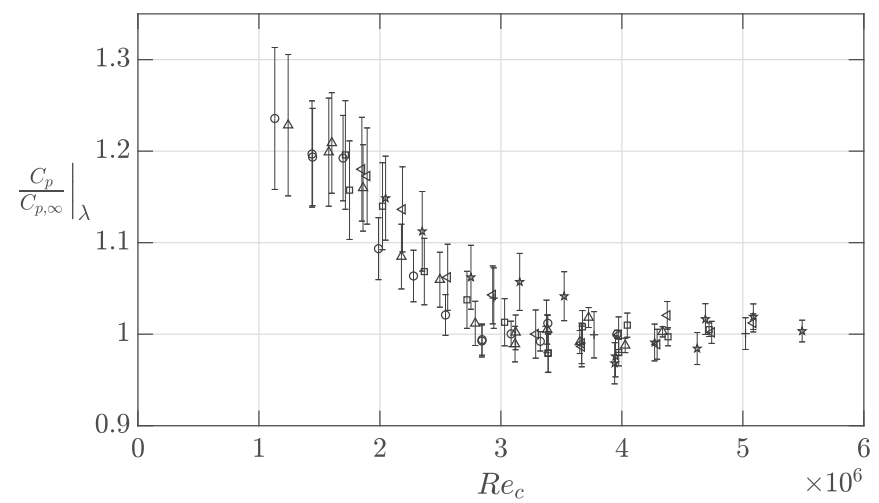

FIG. 8. Power coefficient normalized by the value at Reynolds number invariance for several tip speed ratios. Symbols correspond to $\lambda: 5.0=\circ, 5.5=\triangle, 6.0=\square, 6.5=\triangleleft, 7.0=\star, 7.5=+$. 
for a range of $\operatorname{Re}_{c}$ values gives the resulting $C_{p}$ for any other tip speed. While this specific result is only applicable to the current turbine and test conditions, future work will aim to determine if this is a universal trait of wind turbine operation at high Reynolds numbers.

\section{BOUNDARY LAYER TRANSITION EFFECTS}

Generally, increasing the Reynolds number of a boundary layer will move the point of transition, from laminar to turbulent, upstream. The exact position where the boundary layer transitions is not fully understood, and as such it is challenging to predict. The situation is further complicated on a wind turbine rotor where the boundary layer is experiencing imposed external pressure gradients, streamline curvature, as well as centrifugal forces. Therefore, it is common to pin the transition point using tripping devices near the leading edge with the goal of forcing the boundary layer to be turbulent over nearly all of the airfoil [22,23]. Fundamentally, this has the benefit of eliminating some of the problems that are commonly associated with an airfoil section experiencing transition, such as extreme sensitivity to boundary and initial conditions and laminar separation. Ideally, a tripped airfoil will better approximate the performance of the untripped model tested at very high $\operatorname{Re}$ values, where the boundary layer would naturally transition early on the airfoil surface. Practically, tripping devices are not perfect and typically add some amount of section drag, thus affecting overall performance compared to a naturally transitioning flow.

To determine if the transition is contributing to the somewhat unexpected Reynolds number behavior observed with the untripped rotor, the same rotor model was equipped with tripping devices close to its leading edge. The exact same methodology as was used to study the untripped rotor was otherwise used for the tripped rotor. In the field, effects due to transition might be less significant as debris and other imperfections on the rotor contribute to earlier transition. However, since the transition is highly sensitive to the Reynolds number, and is a phenomenon well known to be challenging to capture numerically, it is important to perform any such studies at field-scale relevant Reynolds numbers.

\section{A. Trip geometry}

Tripping devices were added to the suction and pressure side of the model rotor blade with the goal of fixing the transition location. The trip height, $k_{\mathrm{cr}}$, was determined by using a roughness Reynolds number, $\operatorname{Re}_{k}=600$, as in the work of Braslow and Knox [22]. External velocity was estimated along the rotor span via $U_{\text {ext }}=\sqrt{U^{2}+\omega r^{2}}$, where $U$ is the free-stream velocity and $r$ the local radius. Determination of $k_{\mathrm{cr}}$ was performed at a fixed external tip speed of $\lambda=5.0$ for simplicity and due to the fact that this corresponds to the maximum power coefficient of the untripped rotor for most Reynolds numbers [see Fig. 7(a)]. The value of $k_{\mathrm{cr}}$ along the rotor span is given for a variety of representative $\mathrm{Re}_{D}$ values in Fig. 9.

From this figure, it was determined that a critical roughness height of $k_{\mathrm{cr}} \approx 8 \mu \mathrm{m}$ would be sufficient to trip the rotor at all Reynolds numbers and tip speed ratios. Ideally, the roughness height would vary with $\operatorname{Re}_{D}, \lambda$, and radial location, but this would have created an impractical number of experimental configurations. The main drawback of using a single-sized tripping device is that it can yield additional drag in some cases.

Various methodologies were attempted to produce a reliable and repeatable tripping geometry at the micron scale. Traditional tripping devices such as sand-grain type grit, zigzag tape, and trip wires could not be applied at sufficiently small scale to satisfy the requirements on $k_{\mathrm{cr}}$. The final methodology involved using MEMS-based clean-room tools (West-Bond Incorporated epoxy bonder model 7200A) to manually apply ultraviolet curable epoxy dots (Norland Electronics Adhesive part number 123SBL) to the surface of the rotor. A macrophotograph of the resulting epoxy microdots is shown in Fig. 10.

Following application to both the suction and pressure side of the rotor, a sample of dots was imaged with a confocal microscope (Leica DCM 3D micro-optical system) at various locations on 


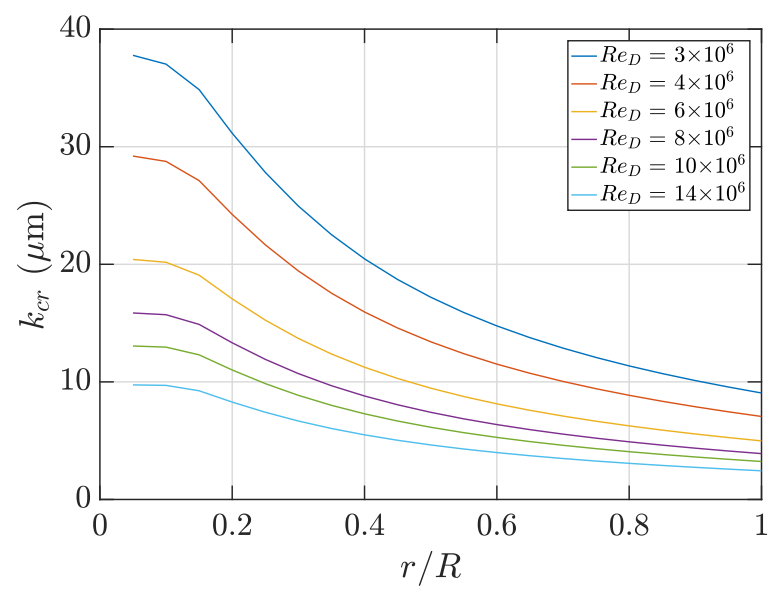

FIG. 9. Critical roughness height for $\mathrm{Re}_{k}=600$ as a function of radial location for various free-stream Reynolds numbers. Note that the external tip speed was fixed at $\lambda=5.0$.

both sides of two blades. The resulting trip height was found to be $k=8.82 \pm 3.95 \mu \mathrm{m}$ for the front row and $k=10.86 \pm 4.11 \mu \mathrm{m}$ for the downstream row. Average diameter was measured to be $184 \mu \mathrm{m}$ with a spacing around $700 \mu \mathrm{m}$. Row spacing was found to be $330 \mu \mathrm{m}$, on average. The dimensions of the trip pattern approximately correspond to classical zigzag tape used in full-scale wind turbine testing.

\section{B. Tripped rotor performance}

Plots of power and thrust coefficients for the tripped rotor are given in Fig. 11. Untripped rotor data from Figs. 6(a) and 6(b) are shown for comparison in the leftmost plots. Note that not all $\mathrm{Re}_{D}$ cases tested for the untripped case are currently available for the tripped rotor. Interestingly, collapse in the power coefficient is seen, within experimental error, over the entire Reynolds number range, with only the lower Reynolds number and tip speed deviating slightly. This result implies that whatever mechanism is causing the performance augmentation at lower $\operatorname{Re}_{D}$ values observed on the untripped rotor is related to, or at least affected by, transition. Interestingly, a more clear Reynolds number trend is observed in the plots of $C_{t}$ than that for the untripped rotor. Higher $\operatorname{Re}_{D}$ values correspond to a larger thrust coefficient for the tripped case, with $\operatorname{Re}_{D}=10 \times 10^{6}$ and $14 \times 10^{6}$ cases collapsing. Data for the tripped rotor were interpolated to a fixed $\lambda$ grid, as previously done for the untripped rotor, to determine the Reynolds number trends in more detail. Figure 12(a) shows the tripped data as a function of $\operatorname{Re}_{c}$. Note that very little change is seen for any tip speed ratio as $\operatorname{Re}_{c}$

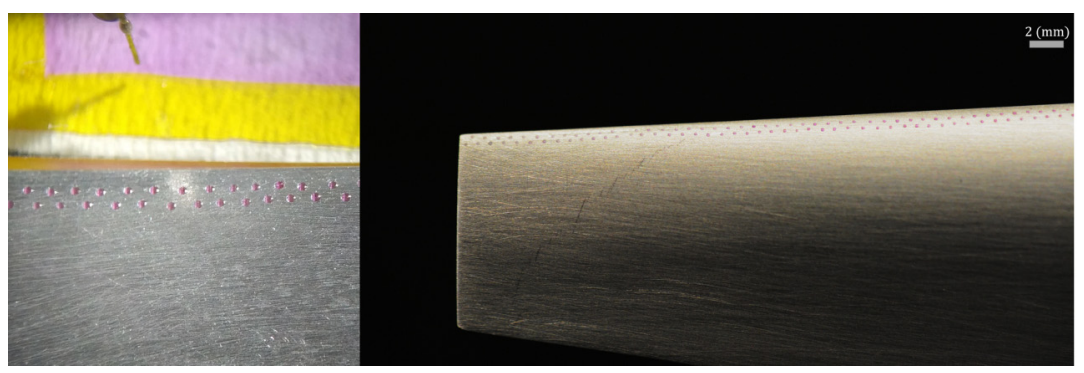

FIG. 10. Dot application process shown on the left, performed via microscope and an epoxy-bonding tool. The right image shows the tip of a finished rotor blade with dots applied. 

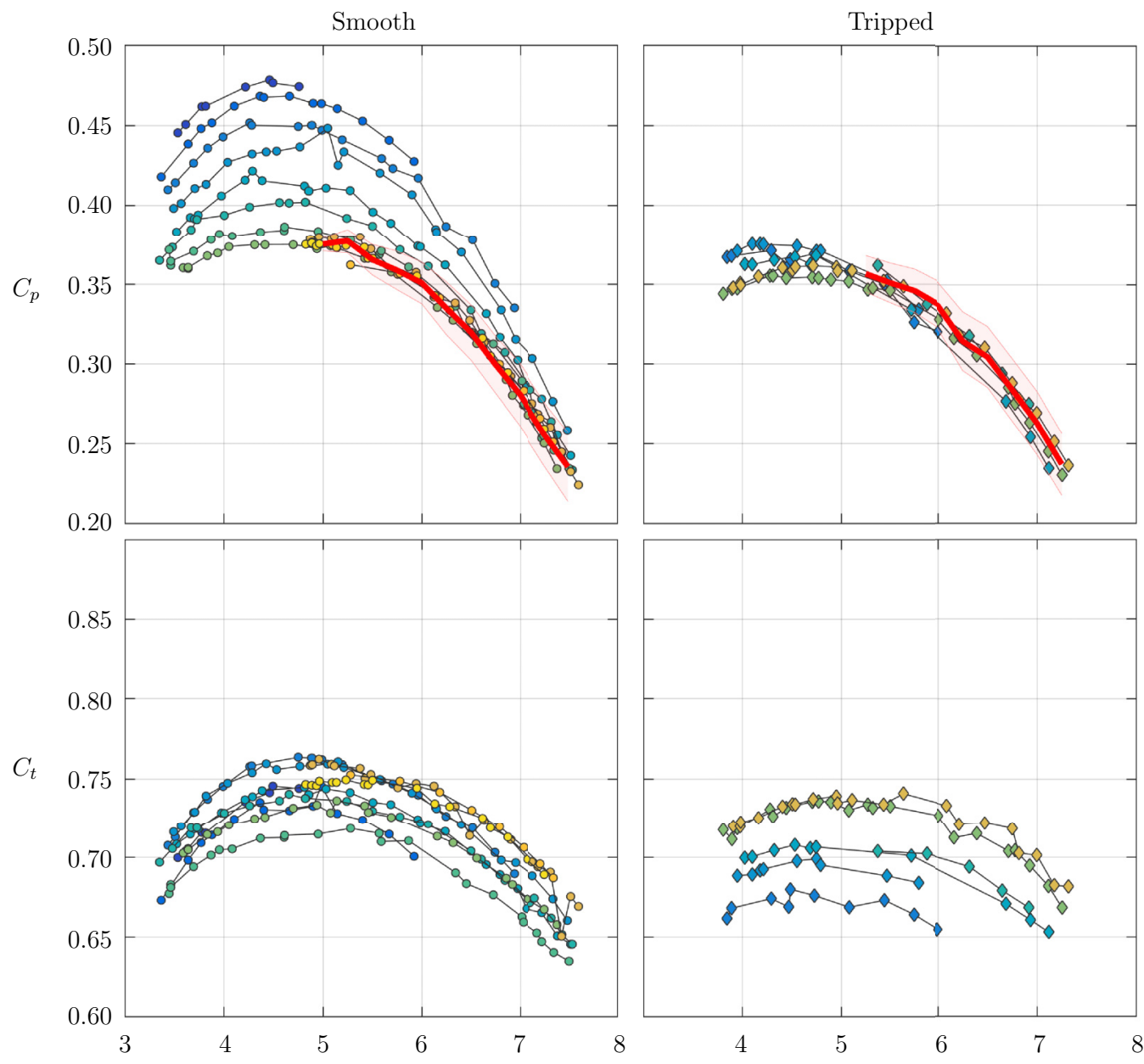

$\lambda$

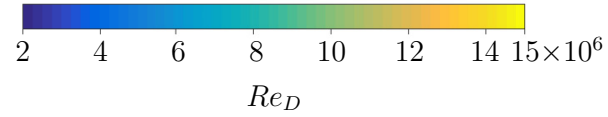

FIG. 11. Power and thrust coefficients for the untripped and tripped rotor geometries. The leftmost plots are reproduced from Fig. 6 for comparison. The red lines represent the respective $C_{p, \infty}$ curve for each rotor case. The value is defined as the mean $C_{p}$ when $\operatorname{Re}_{c} \geqslant 3.5 \times 10^{6}$.

is increased. This confirms the trend observed in the prior figure. It appears that prematurely forcing the boundary layer transition along the rotor surface causes it to perform in a similar manner to the untripped rotor in the high Reynolds number limit. There are slight differences in the behavior, which are more readily observed when viewing the respective $C_{p, \infty}$ values for the tripped versus untripped rotor in Fig. 12(b). It appears that the addition of tripping devices has slightly decreased the high Re performance. This could be due to the additional drag introduced by the trip devices themselves.

Finally, the data of Fig. 12(a) are normalized with the invariant power curves as in the untripped case. The result is given in Fig. 13. As expected, the specific tip speed chosen has little effect on how the power coefficient changes with $\operatorname{Re}_{c}$. When calculating $C_{p, \infty}$ for the tripped case, the restriction 


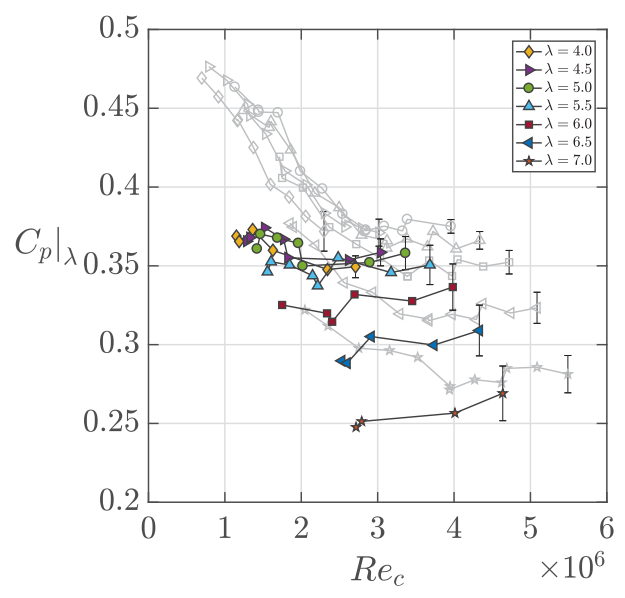

(a)

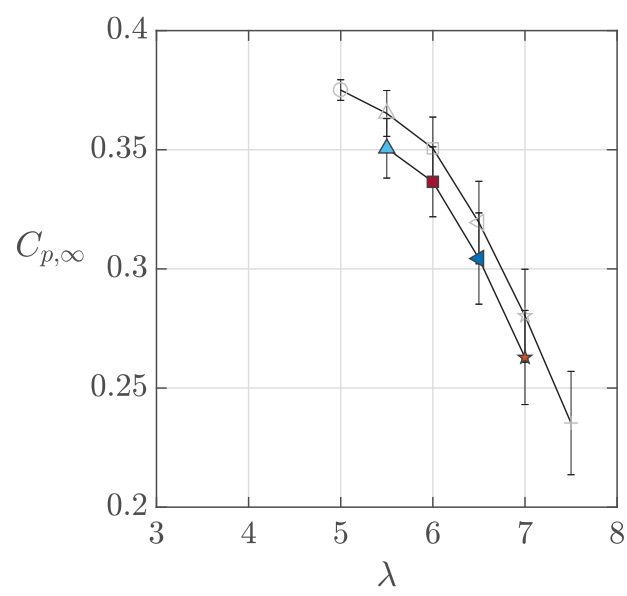

(b)

FIG. 12. Power coefficient as a function of the local blade Reynolds number in (a) for a variety of fixed tip speed ratios, similar to Fig. 7. Part (b) shows the Reynolds number invariant power curve for the model turbine geometry used under laminar inflow conditions. Untripped data points are shown as gray symbols in both plots for reference.

of $\operatorname{Re}_{c} \geqslant 3.5 \times 10^{6}$ could be removed. Invariant behavior is achieved almost immediately, although no $\operatorname{Re}_{c}$ values below 1 million are available to check this behavior at what could be considered conventional laboratory Reynolds numbers.

\section{DISCUSSION OF OBSERVED ROTOR BEHAVIOR}

Two potential mechanisms for the observed rotor behavior are discussed: changes to the 2D lift curve due to thick airfoil sections and sectional drag changes due to surface geometry. The first mechanism is related to a decrease in the lift coefficient with Reynolds number. This method of operation is nonstandard with regard to classic thin-airfoil behavior [23], however a majority of the rotor utilizes airfoils above $20 \%$ thickness ratio, and could be expected to have different behavior from the thin airfoil case. Preliminary measurements have been conducted for two sectional airfoil

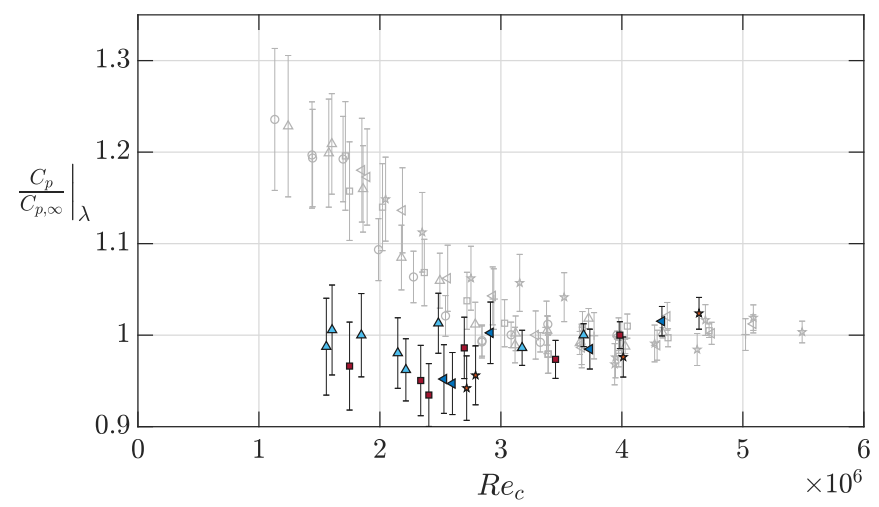

FIG. 13. Power coefficient normalized by $C_{p, \infty}$ for the tripped rotor (color filled symbols) and the untripped rotor data (as gray markers). Symbol shape corresponds to $\lambda: 5.0=0,5.5=\triangle, 6.0=\square, 6.5=\triangleleft, 7.0=\star$, $7.5=+$. 
profiles in order to gain additional insight into their behavior. These are the tip NACA 63-214 (14\% thickness) and the root NACA 63-235 (35\% thickness) airfoils. Several different Reynolds numbers were investigated (on the same order as the fully three-dimensional rotor tests) with the 63-214 profile agreeing well with numerical simulations performed using Mark Drela's XFoil code [24]. The thicker 63-235 airfoil section diverged from typical Reynolds number behavior. Specifically, the lift-curve slope with an angle of attack, $\frac{\partial C_{l}}{\partial \alpha}$, was observed to decrease as the Reynolds number of the airfoil increased. This would indicate a reduced performance of the inner sections with increased $\operatorname{Re}_{c}$ (for a fixed $\alpha$ value) consistent with the observed decrease in rotor performance. These preliminary experiments indicate that the thicker sections of the NACA 6-series may diverge significantly from thin-airfoil theory, with the mechanism causing this divergence still under investigation. It is also not yet clear at what thickness this behavior begins to manifest. Experiments by other authors performed at up to a $21 \%$ thickness ratios using this airfoil family (NACA $63_{3}-221$ section by Abbott and Von Doenhoff [23]) did not display a changing lift curve slope with Reynolds number. Therefore, we expect this transition from classical airfoil behavior to that observed for the thicker 6-series to occur somewhere between these two values. Given that the airfoil section at midradius of the rotor is approximately $26 \%$, a majority of the rotor may be susceptible to these effects.

Another plausible mechanism accounting for the decreased rotor performance is related to the surface features on the rotor itself. In Sec. II A the surface roughness was measured to be $800 \mathrm{~nm}$ in height, on average, across the rotor blade. Although this value is small, the roughness height may be large enough to have some effect if $\mathrm{Re}_{c}$ is made large enough. The complicated nature of surface roughness interactions with the airfoil boundary layer means that a variety of sectional performance changes are possible. In one case, the airfoil boundary layer may be transitioning from a laminar state to turbulence earlier than would be predicted with a numerical code. This would cause additional drag on the rotor, and hence reduce performance, with this effect becoming more pronounced as $\operatorname{Re}_{c}$ is increased until the boundary layer over the airfoil is completely turbulent. This mechanism is supported by the results of Sec. V where the rotor was artificially forced to transition from laminar to turbulent flow near the leading edge, which resulted in decreased, low Re performance that approximated the untripped rotor case operating at high Re.

A second method by which the surface features can affect rotor performance is via aerodynamic roughness. A surface in turbulent flow may become aerodynamically rough if the roughness height becomes larger than the viscous sublayer. In this context, the flow is already turbulent over the airfoil surface, and as Re is increased, the height of the viscous sublayer decreases so that the roughness elements begin to reach beyond the sublayer and affect the buffer region (and potentially beyond) in the boundary layer. This causes an increase in overall section drag as the Reynolds number is increased, until a fully rough regime is achieved where pressure drag on the surface elements dominates viscous drag. In this case, above a particular Reynolds number no additional changes in the drag coefficient of the surface are observed. This type of behavior is evident in a global sense with the power coefficient measurements of the rotor, which are particularly susceptible to changes in drag coefficient.

Both lift reduction (via changes to the lift-curve slope) and drag augmentation (via transition and/or aerodynamic roughness) could be acting on the rotor surface to varying degrees. Additional numerical and experimental work that carefully probes the near-surface flow condition is required to further understand the relevance of each mechanism. The authors do not suspect that rotational effects (or so-called 3D effects) are causing the observed behavior due to the decoupling of $\lambda$ and $\operatorname{Re}_{c}$ possible in this work. If $\lambda$ effects were present, we would expect to see no change as $\operatorname{Re}_{D}$ is altered for a fixed $\lambda$ [see Fig. 7(a)]. The results do highlight how sensitive a design can be to the effects of Reynolds number, even for commonly utilized airfoil families at very large values of $\operatorname{Re}_{c}$.

\section{CONCLUSIONS}

This study presents the highest Reynolds number data currently available for a horizontal axis wind turbine in a laboratory setting. The unique conditions were achieved using a wind tunnel 
that uses highly compressed air as the working fluid. For a rotor with a free transition, Reynolds number effects were clearly seen across a large range of $\operatorname{Re}_{D}$ up to 10 million when considering the peak power coefficient. These observations are surprising given the relatively large values of Re of this rotor, compared to previous work that examined Reynolds number scaling of performance. It is shown that the Reynolds number, and hence scale effects, can alter turbine performance significantly even at relatively large values, although the exact nature of the trends might be a direct function of the specific rotor geometry and airfoils chosen. However, it is likely that a number of designs may show similar performance trends as this rotor uses an airfoil family that is common in the wind industry. Further work is needed to identify the exact mechanism driving the observed trends and to explore the large parameter space of geometric effects in detail.

The ability to control tip speed ratio independently from the Reynolds number is leveraged to gain additional insight into scaling of the performance with the blade Reynolds number, $\operatorname{Re}_{c}$. This has allowed for exploration of Re sensitivity beyond the BEM calculations based on data from individual airfoil sections tested in wind tunnels. With the ability to change model rotational speed accurately, similar to a truly variable speed drive on a full-scale turbine, many different $\lambda$ were tested for fixed $\operatorname{Re}_{D}$ values. The data could then be reinterpreted in terms of the chord-based Reynolds number, which more clearly outlined the scale dependency. For the untripped rotor, a value of $\operatorname{Re}_{c} \geqslant$ $3.5 \times 10^{6}$ was required before Reynolds number invariance was observed in the power coefficient. This cutoff value was independent of the specific $\operatorname{Re}_{D}$ and $\lambda$ operating point, indicating that the performance scaling of this rotor was not driven by rotational effects but by the Reynolds number of the rotor blade itself. This was further tested by renormalizing the power curves by the Reynolds number invariant $C_{p}$ values. In this way, the rotational dependence is removed and all curves showed a similar shape. This result has direct implications for modeling and simulation efforts of rotor performance.

Finally, the effect of transition on rotor performance was investigated in detail by adding carefully prescribed tripping devices to the rotor surface in the form of microdots. With these elements in place, low-Re performance augmentation was eliminated, suggesting that whatever mechanism is causing the increase in $C_{p}$ is related to, or affected by, transition. Future work will investigate this mechanism in additional detail. The results indicate that the boundary layer state is of critical importance to the operation of a turbine, even at relatively high Reynolds numbers.

\section{ACKNOWLEDGMENT}

The support of the National Science Foundation under Grant No. CBET-1652583 (Program Manager Ron Joslin) is gratefully acknowledged.

\section{APPENDIX: CORRECTION METHODOLOGIES}

In the processing of the experimental data sets, two specific corrections were made to account for the effects of drive-train losses inside the gearbox and model blockage in the tunnel. These corrections were standardized and included in the postprocessing of all experimental data sets. The results of Secs. IV and V are therefore the true aerodynamic power produced by the rotor.

\section{Gearbox efficiency correction}

To recover the aerodynamic input power, measured mechanical power from the turbine model was corrected in postprocessing to account for drive-train losses. These inefficiencies mainly stem from the constant meshing of gear teeth inside the turbine gearbox, but they can also be attributed to frictional losses in the bearings and lubricant. To capture these effects, a bulk efficiency of the drive-train is defined as the ratio of output to input power,

$$
\varepsilon=\frac{\left.\tau \omega\right|_{\text {output }}}{\left.\tau \omega\right|_{\text {input }}}
$$




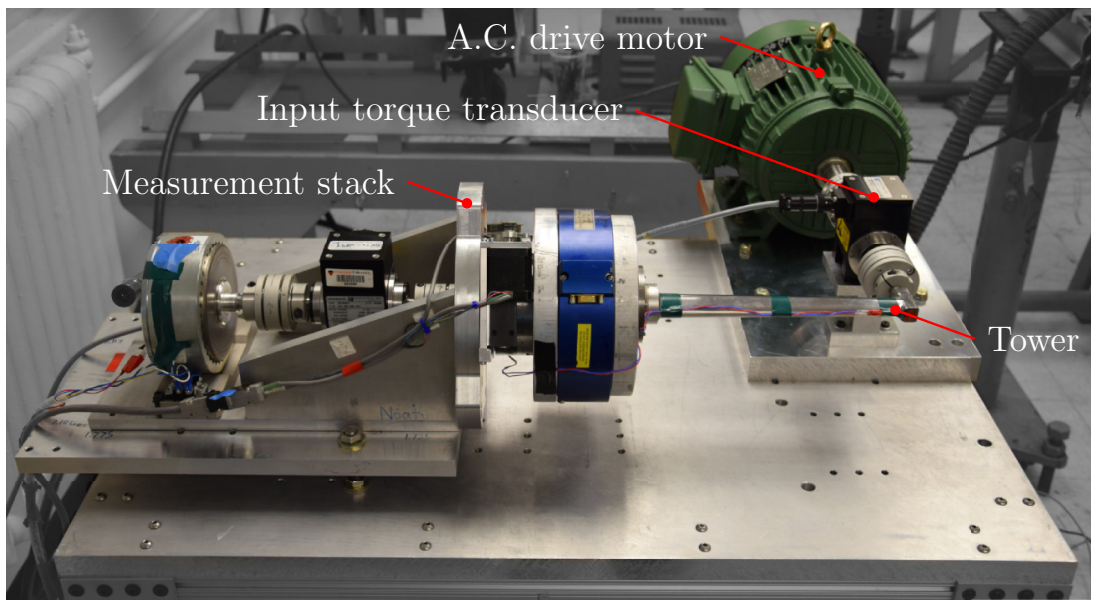

FIG. 14. Test rig shown with measurement stack loaded for efficiency tests.

where $\varepsilon$ is the measured efficiency. Prior to beginning an experimental campaign, $\varepsilon$ was measured with a custom-designed test rig located outside of the HRTF wind tunnel facility. Using this setup, shaft loads representative of those undergone by a wind turbine model could be directly applied to the drive train. The rig itself consists of a $2.24 \mathrm{~kW}$ variable-frequency (ac) motor connected to a torque transducer via a flexible coupling. Input torque and rotational speed output from the motor are measured by the first transducer. Next, in place of the model rotor, a second coupling is attached to the input shaft of the tower gearbox. At this point, the measurement stack is the same as that used during an experiment (described in Sec. II B). This process involves measuring the output power transferred from the tower to the second torque transducer. Power is then removed via the magnetic hysteresis brake. The main benefit of this setup is that the entire measurement stack as used during an experiment (and as shown in Fig. 3) is used for measuring $\varepsilon$. The test rig with the measurement stack mounted in place is shown in Fig. 14. Measurements of $\varepsilon$ are completed at over 175 different operating points (torque and speed settings) representative of those encountered inside the HRTF. The resulting efficiency map is shown in Fig. 15. The individual test points for $\varepsilon$ are shown as black

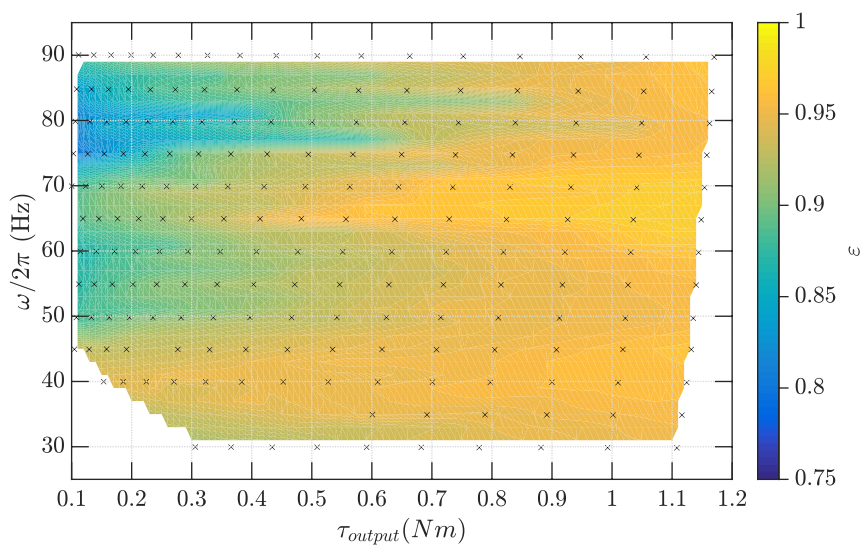

FIG. 15. Efficiency map as measured on the test rig. Black crosses represent individual test points where $\varepsilon$ was measured. To aid in visualizing the efficiency and its dependence on both $\tau$ and $\omega$, a color contour map was generated from the data at these test points with the magnitude given by the color bar at right. 


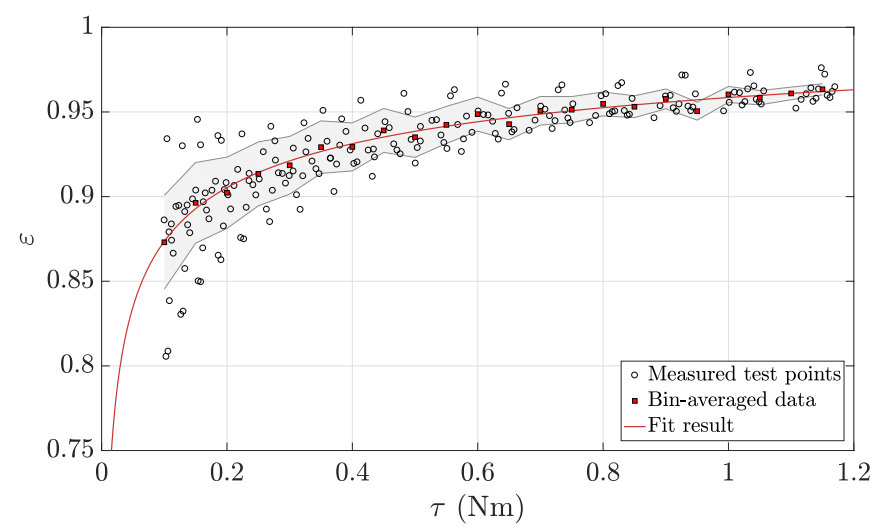

FIG. 16. Measured gearbox efficiency as a function of the input torque only. Also shown are the binaveraged data and resulting curve fit using Eq. (A2) implemented to correct experimental data.

dots, and the contour has been generated to aid in visualizing the dependency of the efficiency on $\omega$ and $\tau$. A sharp drop is seen in $\varepsilon$ when $\tau_{\text {output }} \leqslant 0.1(\mathrm{Nm})$. This indicates that below some loading threshold, the uncertainty in measured efficiency is large due to the steep slope in the efficiency curve. In contrast, very little dependency is seen for $\omega$. Only small variations are seen across the range of speeds for a fixed output torque. The exception to this case is when $\omega / 2 \pi \leqslant 40$, where fewer data are available. However, experiments are rarely performed in this regime, and so for the bulk of the operating space of the turbine, speed dependence is negligible.

A functional and simple correction methodology was desired so that mapping the measured power to the true aerodynamic power would be a straightforward and repeatable process. Given the results shown in Fig. 15, a fit of the form $\varepsilon=f(\tau)$ was sought, with error bars that are proportional to the scatter in $\omega$. A power-law relationship was found to capture the changes in efficiency with torque:

$$
\varepsilon=a\left(\tau_{\text {output }}\right)^{b}+c
$$

where $a, b$, and $c$ are adjustable constants determined by a best fit of the measured efficiency from the test rig. Since the dependency on $\omega$ has been neglected, data were bin-averaged by $\tau$ across all speeds in steps of $\Delta \tau=0.05(\mathrm{Nm})$ over $\tau \in[0.10,1.2]$. The standard deviation of the resulting binaveraged value of $\varepsilon$ gives an estimate of the error associated with neglecting the speed dependency. The results of this method are shown in Fig. 16. Above a minimum applied torque (with a small functional dependence on operating speed) the gearbox efficiency exceeded $90 \%$, which compares well to the value of $\varepsilon=98 \%$ found in the literature for right-angle bevel gearboxes [25,26]. It is interesting to note the sharp drop off in efficiency at low torque values in Fig. 15, supporting the idea that a gearbox of this type needs some minimum loading in order to operate efficiently.

\section{Model blockage correction}

Flow acceleration due to the presence of a model and constraining walls affects all wind tunnel experiments to a varying degree. For turbine and propeller studies, the method of Glauert [5] is typically used to correct the free-stream velocity (see, for example, Refs. [27,28]). This methodology has been applied to the current experiments by utilizing the thrust coefficient (as given by the axial force measured at the tower base) and the known geometric blockage of the turbine. An iterative solver is implemented to find the corresponding equivalent free air speed, $U$. This is the free-stream velocity that gives the same thrust for a corresponding rotor velocity as the model operating in the tunnel and is used in place of the tunnel velocity, $U_{t}$, for all experimentally determined values. 


\section{a. Tower drag effects on blockage}

The axial thrust force is recorded by the six-axis load cell located at the base of the turbine and therefore the reported thrust coefficient values include the axial force generated by the turbine plus drag due to the tower assembly. The magnitude of this effect can be estimated if it is assumed that the rotor thrust and tower drag are linear so that the measured thrust is $F_{t}=F_{\text {rotor }}+F_{\text {tower }}$, and the thrust coefficient becomes

$$
C_{t}=\frac{F_{\text {rotor }}+F_{\text {tower }}}{\frac{1}{2} \rho U^{2} A_{\text {rotor }}}=C_{t, \text { rotor }}+C_{d, \text { tower }} \frac{A_{\text {tower }}}{A_{\text {rotor }}},
$$

where $C_{d \text {,tower }}$ is the drag coefficient of the tower geometry and $A_{\text {tower }}$ is the frontal area of the tower which characterizes this drag. For a cylinder at moderate Reynolds numbers, this drag coefficient could be as large as 0.5 but will decrease as Re of the tower increases. In the case of the HAWT model tower, a more aerodynamic diamond-shaped profile was used. For these experiments, the drag coefficient of the tower was not measured directly as a function of Reynolds numbers, but estimates can be made of their effect using Eq. (A3) and an assumed value for $C_{d \text {,tower }}$. For the total frontal area of the rotor:

$$
\frac{A_{\text {tower }}}{A_{\text {rotor }}}=0.161 \text {. }
$$

For a worst-case scenario of $C_{d \text {,tower }}=0.5$, the increase in measured thrust coefficient would be 0.081 , or using a more realistic value of $C_{d \text {,tower }}=0.1$ returns a change in $C_{t}$ of 0.0161 , which is nominally near the sensor resolution. For all of the data reported here, no correction for tower drag was made to any of the reported thrust coefficient values for several reasons. The first is that the specific value of $C_{d \text {,tower }}$ is in reality difficult to separate from the measured $C_{t}$,rotor because the two interact in a complicated, and likely nonlinear fashion with Reynolds number. The prior discussion also assumed a single velocity scale determined the tower drag, but a significant portion of the tower is downstream of the rotor, meaning that the resulting drag force could be much less than expected. Therefore, any corrections applied to the thrust coefficients for the tower drag would need to make a number of assumptions about the way the drag varies across the tower and with Re. Ideally, an alternative method would be utilized to measure the rotor thrust, but this is not feasible with the current setup.

[1] J. Cotrell, T. Stehly, J. Johnson, J. O. Roberts, Z. Parker, G. Scott, and D. Heimiller, Analysis of transportation and logistics challenges affecting the deployment of larger wind turbines: Summary of results, Tech. Rep. TP-5000-61063, National Renewable Energy Lab, Golden, CO, 2014.

[2] T. G. Herges, D. C. Maniaci, B. T. Naughton, T. Mikkelsen, and M. Sjöholm, High resolution wind turbine wake measurements with a scanning lidar, J. Phys.: Conf. Ser. 854, 012021 (2017).

[3] D. Simms, S. Schreck, M. Hand, and L. J. Fingersh, NREL unsteady aerodynamics experiment in the NASA-Ames wind tunnel: A comparison of predictions to measurements, Report TP-500-29494, National Renewable Energy Lab, Golden, CO, 2001.

[4] Power Performance of Electricity-Producing Wind Turbines Based on Nacelle Anemometry, Tech. Rep. IEC 61400-12, International Electrotechnical Commission, 2017.

[5] H. Glauert, Airplane propellers, in Aerodynamic Theory (Springer, Berlin, 1935), pp. 169-360.

[6] H. A. Madsen, R. Mikkelsen, S. Øye, C. Bak, and J. Johansen, A detailed investigation of the blade element momentum (BEM) model based on analytical and numerical results and proposal for modifications of the BEM model, J. Phys.: Conf. Ser. 75, 012016 (2007).

[7] G. A. M. van Kuik, J. Peinke, R. Nijssen, D. Lekou, J. Mann, J. N. Sørensen, C. Ferreira, J. W. van Wingerden, D. Schlipf, P. Gebraad, H. Polinder, A. Abrahamsen, G. J. W. van Bussel, J. D. Sørensen, P. Tavner, C. L. Bottasso, M. Muskulus, D. Matha, H. J. Lindeboom, S. Degraer, O. Kramer, S. Lehnhoff, 
M. Sonnenschein, P. E. Sørensen, R. W. Künneke, P. E. Morthorst, and K. Skytte, Long-term research challenges in wind energy — a research agenda by the European Academy of Wind Energy, Wind Energ. Sci. 1, 1 (2016).

[8] L. Vermeer, J. Sørensen, and A. Crespo, Wind turbine wake aerodynamics, Progr. Aerospace Sci. 39, 467 (2003).

[9] J. Schepers and H. Snel, Model experiments in controlled conditions, Report ENC-E-07-042, ECN, 2007.

[10] J. G. Schepers, K. Boorsma, A. Bon, C. Kim, and T. Cho, Results from Mexnext: Analysis of detailed aerodynamic measurements on a $4.5 \mathrm{~m}$ diameter rotor placed in the large German Dutch Wind tunnel DNW, Tech. Rep. ECN-M-11-034, Energy Research Center of the Netherlands, Petten, NL, 2011.

[11] S. Schreck, T. Sant, and D. Micallef, Rotational Augmentation Disparities in the MEXICO and UAE Phase VI Experiments, Conference Paper NREL/CP-500-47759 (https://www.nrel.gov/docs/fy10osti/47759.pdf, 2010).

[12] J. M. Jiménez, J. J. Allen, and A. Smits, Preliminary velocity measurements in the wake of a submarine model, DLR-Mitteilung No. 3, 1229 (2001).

[13] J. M. Jiménez, M. Hultmark, and A. J. Smits, The intermediate wake of a body of revolution at high Reynolds numbers, J. Fluid Mech. 659, 516 (2010).

[14] A. Ashok, T. Van Buren, and A. Smits, Asymmetries in the wake of a submarine model in pitch, J. Fluid Mech. 774, 416 (2015).

[15] M. Vallikivi, M. Hultmark, and A. J. Smits, Turbulent boundary layer statistics at very high Reynolds number, J. Fluid Mech. 779, 371 (2015).

[16] M. A. Miller, J. Kiefer, C. Westergaard, and M. Hultmark, Model wind turbines tested at full-scale similarity, J. Phys.: Conf. Ser. 753, 032018 (2016).

[17] M. A. Miller, S. Duvvuri, I. Brownstein, M. Lee, J. O. Dabiri, and M. Hultmark, Vertical axis wind turbine experiments at full dynamic similarity, J. Fluid Mech. 844, 707 (2018).

[18] M. A. Miller, S. Duvvuri, W. D. Kelly, and M. Hultmark, Rotor solidity effects on the performance of vertical-axis wind turbines at high Reynolds numbers, J. Phys. Conf. Ser. 1037, 052015 (2018).

[19] M. V. Zagarola, Mean-flow scaling of turbulent pipe flow, Ph.D. thesis, Princeton University, 1996.

[20] R. M. Reynolds, Preliminary results of an investigation of the effects of spinner shape on the characteristics of an NACA D-type cowl behind a three-blade propeller, including the characteristics of the propeller at negative thrust, Tech. Rep. NACA RM A53J02, DTIC Document, 1953.

[21] M. O. L. Hansen, Aerodynamics of Wind Turbines, 3rd ed. (Earthscan, London, UK, 2007), Vol. 17.

[22] A. L. Braslow and E. C. Knox, Simplified method for determination of critical height of distributed roughness particles for a boundary layer transition at mach numbers from 0 to 5, Tech. Rep. 4363, National Advisory Committee for Aeronautics, Langley, VA, 1958.

[23] I. H. Abbott and A. E. Von Doenhoff, Theory of Wing Sections, Including a Summary of Airfoil Data (Courier, New York, NY, 1959).

[24] M. Drela, in XFOIL: An analysis and design system for low Reynolds number airfoils, Low Reynolds Number Aerodynamics, Lecture Notes in Engineering, Vol. 54, edited by T. J. Mueller (Springer, Berlin, Heidelberg, 1989).

[25] S. P. Radzevich and D. W. Dudley, Handbook of Practical Gear Design (CRC, Boca Raton, FL, 1994).

[26] J. E. Shigley, R. G. Budynas, and C. R. Mischke, Mechanical Engineering Design (McGraw-Hill, New York, NY, 2004).

[27] R. F. Mikkelsen, Actuator disk methods applied to wind turbines, Ph.D. thesis, Technical University of Denmark, 2004.

[28] A. S. Bahaj, A. F. Molland, J. R. Chaplin, and W. M. J. Batten, Power and thrust measurements of marine current turbines under various hydrodynamic flow conditions in a cavitation tunnel and a towing tank, Renew. Energy 32, 407 (2007). 